\title{
Quantum Ising model in a transverse random field: A density-matrix renormalization group analysis
}

\author{
A. Juozapavičius, S. Caprara, \\ Department of Theoretical Physics, Royal Institute of Technology, SE-100 44 Stockholm, Sweden
}

The spin-1/2 quantum Ising chain in a transverse random magnetic field is studied by means of the density-matrix renormalization group. The system evolves from an ordered to a paramagnetic state as the amplitude of the random field is increased. The dependence of the magnetization on a uniform magnetic field in the $z$ direction and the spontaneous magnetization as a function of the amplitude of the transverse random magnetic field are determined. The behavior of the spin-spin correlation function both above and at criticality is studied. The scaling laws for magnetization and correlation functions are tested against previous numerical and renormalization-group results.

PACS numbers: 75.40.Mg, 75.10.Jm, 05.50.+q

\section{INTRODUCTION}

The spin- $1 / 2$ quantum Ising model in a transverse random magnetic field (QIMRF) has been the subject of an increasing interest in recent times. The main property of the QIMRF is that it undergoes a disorder-driven quantum phase transition in the ground state. Moreover, the critical point of the QIMRF is not an isolated point of nonanalyticity, and the zero-field susceptibility diverges in a wide region around the critical point. This behavior is due to the existence of diluted domains of the wrong phase in the system. Such domains are stabilized by the disorder. This nonanalytical behavior is much more pronounced in the quantum model than in its classical counterpart, pointing out the difference between quantum and thermal fluctuations in disordered systems. More recently the QIMRF has been obtained as an effective model for the one-dimensional Kondo lattice modell (KLM) by means of bosonization of the conduction electrons. The critical behavior of the KLM has indeed features which could be explained in terms of the corresponding QIMRF

Previous numerical results 3 exploited the mapping of the QIMRF in zero external uniform field onto a model of noninteracting fermions. This mapping allows for a detailed study of correlation functions, but is limited by the maximum size of the system that can be handled. On the other hand, in the presence of an external uniform field in the $z$ direction the model is not solvable. In this paper we make use of the density-matrix renormalization-group (DMRG) approach in its infinitesize version to overcome the limitations imposed by the mapping to free fermions. By explicitly introducing an external uniform field we are able to study the magnetization and extrapolate the spontaneous magnetization, thus testing the scaling results and determining the corrections beyond scaling. Moreover our computing time depends but linearly on the size of the system, for a given accuracy.

In this paper we study the spin-1/2 QIMRF defined by the Hamiltonian

$$
\mathcal{H}=-J \sum_{i} S_{i}^{z} S_{i+1}^{z}-\sum_{i} h_{i} S_{i}^{x}-h_{z} \sum_{i} S_{i}^{z}
$$

where the $S_{i}^{\alpha}=\sigma_{i}^{\alpha} / 2,\left\{\sigma_{i}^{\alpha}\right\}$ are Pauli spin matrices, $h_{i}$ are random-field values uniformly distributed in the interval $-h_{0}<h_{i}<h_{0}$, and $h_{z}$ is a constant field in the $z$ direction, added to produce spontaneous symmetry breaking in our subsequent calculations. We take the units of energy such that $J=1$. For the sake of definiteness we study the ferromagnetic case $J>0$, to which the antiferromagnetic model can be reduced by a gauge transformation on every second site $\tilde{S}_{i}^{z}=(-1)^{i} S_{i}^{z}, i$ being the site index. Obviously in the antiferromagnetic case $h_{z}$ has to be taken as a staggered field. Moreover, a gauge transformation $\tilde{h}_{i}=\operatorname{sgn}\left(h_{i}\right) h_{i}, \tilde{S}_{i}^{x}=\operatorname{sgn}\left(h_{i}\right) S_{i}^{x}$ can be performed to make all $h_{i}$ positive.

\section{PREVIOUSLY KNOWN ANALYTICAL RESULTS}

The QIMRF was studied analytically by McCoy and Wu, 1 Shankar and Murthy, 5 and Fisher. 6 Fisher, in particular, makes use of the renormalization group, to determine the critical properties of the model defined by the Hamiltonian

$$
\mathcal{H}^{F}=-\sum_{i} J_{i}^{F} \sigma_{i}^{z} \sigma_{i+1}^{z}-\sum_{i} h_{i}^{F} \sigma_{i}^{x}-H \sum_{i} \sigma_{i}^{z}
$$

with all $J_{i}^{F}$ and $h_{i}^{F}$ positive random variables, drawn independently from two distributions of densities $\pi(J), \rho(h)$.

The phase transition is found to occur at the point where $\overline{\ln h^{F}}=\overline{\ln J^{F}}$, where the bar denotes average over disorder. The distance from criticality is defined as

$$
\delta \equiv \frac{\overline{\ln h^{F}}-\overline{\ln J^{F}}}{\operatorname{var}\left(\ln h^{F}\right)+\operatorname{var}\left(\ln J^{F}\right)} .
$$

In Fisher's notation, the distributions chosen in this paper are 


$$
\pi(J)=\delta(J-1), \quad \rho(h)= \begin{cases}h_{0}^{-1} & \text { for } 0<h<h_{0}, \\ 0 & \text { otherwise }\end{cases}
$$

The relations between our and Fisher's variables are $J=$ $4 J_{i}^{F}, h_{i}=2 h_{i}^{F}, h_{z}=2 H$.

Both in the ordered $(\delta<0)$ and in the disordered $(\delta>0)$ phase a Griffiths ${ }^{-}$region does exist in which the magnetization is nonanalytical at $H=0$. The weakly ordered Griffiths "phase" extends over the region where $\min \left\{J_{i}^{F}\right\}<\max \left\{h_{j}^{F}\right\}, \delta<0$. The weakly disordered Griffiths "phase" extends over the region where $\max \left\{J_{i}^{F}\right\}>\min \left\{h_{j}^{F}\right\}, \delta>0$.

Fisher has found the exact critical scaling function of the magnetization and he showed in particular that the magnetization at the critical point behaves as

$$
M(\delta=0, H) \sim\left[\ln \left(D_{h} / H\right)\right]^{\phi-2},
$$

where $\phi=(1+\sqrt{5}) / 2=1.618033 \ldots$ is the golden mean and $D_{h}$ is some nonuniversal scale factor. The spontaneous magnetization in the ordered phase depends on the distance from criticality according to

$$
M(\delta<0, H=0) \sim(-\delta)^{2-\phi},
$$

for $\delta \rightarrow 0^{-}$. Further away from the critical point, in the weakly ordered phase $(\delta<0)$, for small $H$

$$
M(H)-M_{0} \sim(H)^{1 /(1+z)}[\ln (D / H)]^{x},
$$

where $M_{0}$ is the spontaneous magnetization, the exponent $x$ cannot be determined within renormalization group, $D$ is a nonuniversal scale factor, and

$$
z \approx \frac{1}{2|\delta|}
$$

In the weakly disordered phase $(\delta>0)$, instead

$$
M(H) \sim \delta^{3-\phi}(H)^{1 / z}\left[\ln \left(D^{\prime} / H\right)\right]^{1+1 / z},
$$

where $D^{\prime}$ is another nonuniversal scale factor, and $H \ll$ $D^{\prime}$.

The two-point spin-spin correlation function

$$
C(r)=\left\langle\sigma_{0}^{z} \sigma_{r}^{z}\right\rangle
$$

has the typical behavior

$$
-\ln C(r) \sim \sqrt{r}
$$

at the critical point $\delta=0$ in the limit of large $r$. The $\langle\cdots\rangle$ denotes the quantum average in the ground state. In the disordered phase $\delta>0, C(r)$ should obey the scaling equation

$$
\overline{\ln \frac{C(r ; \delta)}{C(r ; \delta=0)}}=\ln \tilde{C}(r / \tilde{\xi}) \approx-r / \tilde{\xi},
$$

for $r \gg \tilde{\xi}$, where the typical correlation length $\tilde{\xi}$ has the behavior

$$
\tilde{\xi} \sim \frac{1}{\delta^{\tilde{\nu}}},
$$

with $\tilde{\nu}=1$. Young and Rieger model in Eq. (1) for $h_{z}=0$ onto a model of free fermions, numerically calculated the typical correlation functions on finite-size chains. They obtain that their data scale best for $\tilde{\nu}=1.1$.

\section{DMRG ALGORITHM}

The DMRG algorithm introduced by White is used with minor changes. The random field breaks the symmetry of the chain under reflection and separate density matrices are needed for the left and the right subsystems. At each step of renormalization a new block is defined by truncating the Hilbert space of the left and right subsystems onto the eigenstates of the density matrix of the subsystem which correspond to the largest eigenvalues. In our calculation the minimum number of states kept is 8 at each iteration step. However, if a degeneracy occurs in the density matrix, then all the degenerate eigenstates are kept, a procedure which significantly decreases the truncation errorl. Even at the point of the phase transition we found a relative truncation error of the order $10^{-10}$, which makes our results very accurate.

The DMRG algorithm is used to calculate the average magnetization of the chain and the spin-spin correlation functions at some given value of the amplitude of the random field. In the first case the average of the $z$ component of the spin operator of the full system is calculated after 50 DMRG steps (the system is then composed of 102 sites). The value is averaged over a large number (usually 1000, sometimes up to 6000 , depending on the values of $h_{z}$ ) of different random-field configurations. In the second case $N=20-40$ DMRG steps are ignored to make the system large enough so that the edges would have a small influence on the innermost spins. One spin in the middle of the lattice is marked $a$ and the matrix of its $z$ component is $S_{a}^{z}$ at the step $N+1$. The chain can be represented as $B_{L}$ as $B_{R}$, where $B_{L}$ denotes the left block of spins, $B_{R}$ the right block and $s$ is another spin site in the middle of the lattice. The correlation function of the $a$ spin with itself is trivially calculated as $C(0)=\left\langle\delta_{L} \otimes\left(S_{a}^{z}\right)^{2} \otimes \delta_{s} \otimes \delta_{R}\right\rangle=1 / 4$. The $\delta$ 's are unit matrices of the corresponding parts of the system. At the step $N+2$ the left subsystem $B_{L} a$ is renormalized defining a new block $B_{L}^{\prime}$, according to White's procedure ${ }^{3}$ in our procedure we cannot, however, exploit the symmetry of the system under reflection, so that the right subsystem has to be renormalized separately, yielding a new block $B_{R}^{\prime}$. Finally two more sites are inserted between the new blocks. The system looks now like $B_{L}^{\prime} b s B_{R}^{\prime}$, where the left one of the two new sites is called $b$. The spin $a$ is now the rightmost spin in the block $B_{L}^{\prime}$, so that its spin matrix is no longer equal to the simple $2 \times 2$ spin matrix, but it is some matrix $S_{L}^{z}(1)$ of dimension $m \times m$, 
obtained by truncating the product $\delta_{L} \otimes S_{a}^{z}$. Here $m$ is the number of states retained in the block at each step ( 8 or more). The number 1 in the parentheses denotes the distance of the spin $a$ from the right edge of the $B_{L}$. The correlation function between the spins $a$ and $b$ is $C(1)=\left\langle S_{L}^{z}(1) \otimes S_{b}^{z} \otimes \delta_{s} \otimes \delta_{B}^{\prime}\right\rangle$. After each RG step the spin $a$ "moves" deeper into the block $B_{L}$ and a correlation function between spins that are farther apart is calculated according to $C(r)=\left\langle S_{L}^{z}(r) \otimes S_{b}^{z} \otimes \delta_{s} \otimes \delta_{B}^{\prime}\right\rangle$. We repeat the procedure until a sufficient number (50) of values of $C(r)$ is obtained. All of them are averaged over a large number of random field configurations (3000-8000).

It should be mentioned that the magnetization must be calculated at nonzero (though very small) $h_{z}$ to have spontaneous symmetry breaking. This small additional field makes the magnetization larger than the spontaneous magnetization $M_{0}=M\left(h_{z}=0\right)$. Several runs at different $h_{z}$ are needed to extrapolate $M_{0}$. We shall return to this point later. On the other hand, as usual, the correlation functions are directly calculated at $h_{z}=0$.

\section{NUMERICAL RESULTS AND DISCUSSION}

We carefully tested the reliability and accuracy of our algorithm in the two exactly solvable limits of Hamiltonian (11), i.e., $h_{0}=0$ (pure Ising model) and $J=0$ (noninteracting spins in a random field). In the first case we reproduced the exact ground-state wavefunction $|\Phi\rangle=\prod_{i}\left|\uparrow_{i}\right\rangle$ (for $h_{z} \rightarrow 0^{+}$), the ground-state energy $E_{0}=-J(N-1)$, where $N$ is the number of sites, and the correlation function $C(r)=1 / 4, \forall r$. In the second case we checked that for a given random-field configuration, the ground-state wavefunction is $|\Phi\rangle=$ $2^{-N / 2} \prod_{i}\left[\left|\uparrow_{i}\right\rangle+\operatorname{sgn}\left(h_{i}\right)\left|\downarrow_{i}\right\rangle\right]$ with a ground-state energy $E_{0}=-1 / 2 \sum_{i}\left|h_{i}\right|$. We also reproduced the correct average of the ground-state energy and correlation functions over disorder.

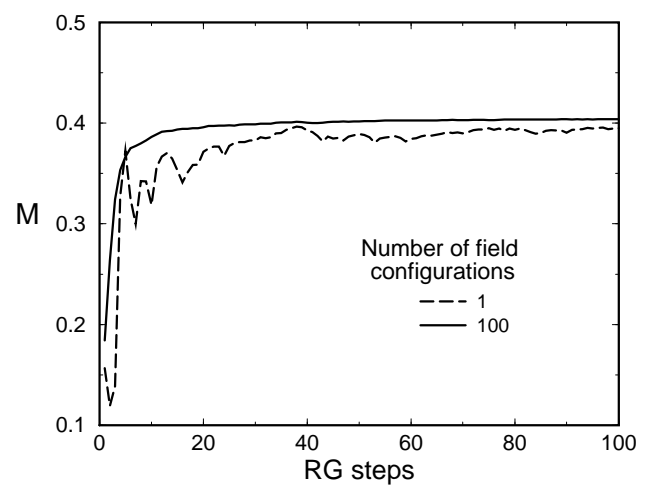

FIG. 1. The magnetization dependence on the system size. All our further calculations are interrupted after $50 \mathrm{RG}$ steps, when the magnetization has saturated.

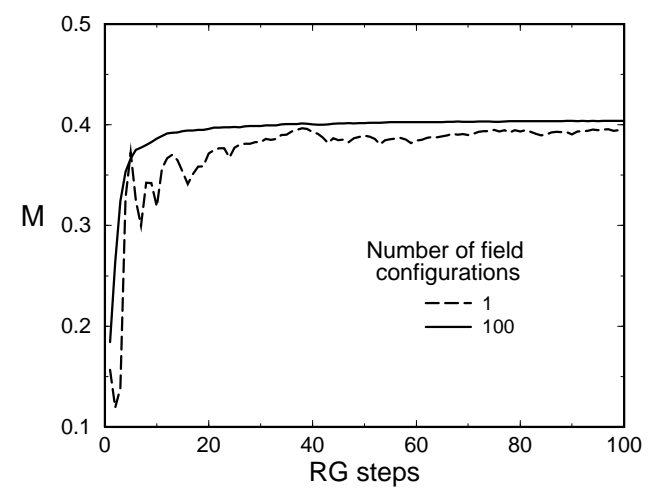

FIG. 2. The dependence of the magnetization $M$ and the estimated error of the mean $\sigma_{\mu}$ on the number of random magnetic field configurations $N_{c}$. Here $\sigma_{\mu}=\sqrt{\sum_{i}\left(M_{i}-\bar{M}\right)^{2}} / \sqrt{N_{c}\left(N_{c}-1\right)}$, where a bar denotes the average over configurations.

The dependence of the calculated quantities both on system size (i.e., on the number of RG steps) and on the number of random-field configurations has to be carefully discussed. A sufficiently large number of RG steps should be performed to eliminate the effects of the edges of the lattice. As is seen in Fig. 1, the magnetization is an increasing function of the size of the chain. The effect in this case becomes typically negligible after 50 RG steps. Figure 2 shows that oscillations of the magnetization as well as the estimated error of the mean decrease quite rapidly with increasing number of field configurations. Usually 1000 configurations are enough for the relative error to become $1-2 \%$ though a larger number of them is required at small external fields. Again we point out that in all our calculations the relative truncation error was of the order $10^{-10}$, so that the errors that affect our data are purely statistical, the convergence to the infinite-size limit being controlled by the number of RG steps, and the accuracy of our averages being determined by the number of random-field configurations.

The magnetization dependence on the amplitude of the random magnetic field $h_{0}$ at $h_{z}=1 \times 10^{-5}$ is shown in Fig. 3. The system is in a ferromagnetic state at small fields and $M=1 / 2$. The magnetization decreases with increasing $h_{0}$ and the system is driven towards a paramagnetic state. Equation (3) predicts the phase transition to occur at $h_{0}=e / 2=1.3591 \ldots$ since the distribution (㺼) gives in our case

$$
\delta=\ln \frac{2 h_{0}}{e},
$$

once the mapping to Fisher's variables is taken into account. The critical point is fairly well reproduced by our extrapolation. 


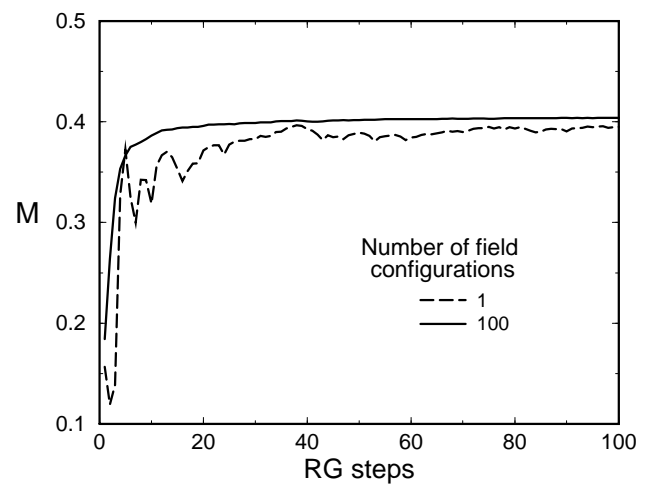

FIG. 3. The magnetization dependence on the amplitude of the random magnetic field at the small ordering field $H=h_{z} / 2=5 \times 10^{-6}$ and $H=0$. The error bars are smaller than the symbols. The data are averaged over 1000 samples. The solid line is a fit according to Eq. (6).

The spontaneous magnetization $M\left(h_{z}=0\right)$ decreases according to Eq. (6). The solid line is the best fit of the form $M_{0}=a(-\delta)^{\beta}$ with $a=0.56 \pm 0.02$ and $\beta=0.40 \pm$ 0.02 . The exponent $\beta$ agrees well with the predicted value $2-\phi=(3-\sqrt{5}) / 2 \approx 0.382$. The values of $M_{0}$ where obtained by fitting Eq. (7). In our fit we find that the exponent $x$ is negligibly small.

The magnetization as a function of the external uniform field at the critical point is plotted in Fig. 4. The result agrees with Eq. (5). The solid line is a fit of the form $M(H)=c\left[\ln \left(D_{h} / H\right)\right]^{\phi-2}$ with $c=0.34 \pm 0.01$ and $D_{h}=0.024 \pm 0.005$.

We also addressed the issue of determining the deviations of the exponent $z$ from its scaling value $1 /(2|\delta|)$. By fitting Eq. (7) and Eq. (9) for $\delta<0$ and $\delta>0$, respectively, we obtained the $1 / z$ as a function of $2|\delta|$, plotted in Fig. 5. The exponent $z$ is smaller than its scaling value in the ordered phase, and larger in the disordered phase.

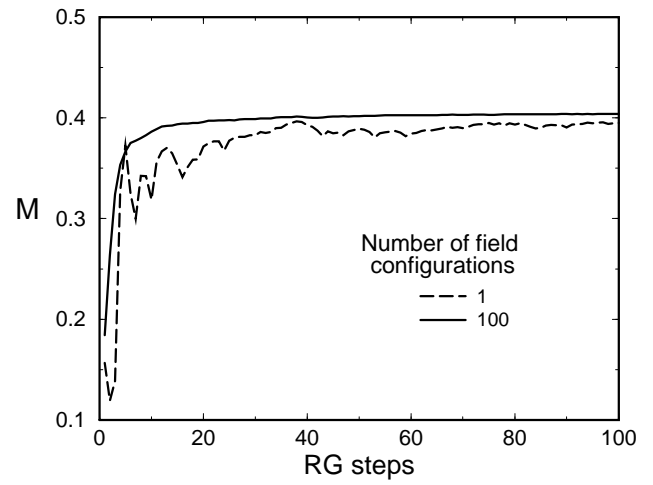

FIG. 4. The magnetization dependence on the ordering field at criticality. The solid line is a fit of the form (5).

The average of the logarithm of the two-point spinspin correlation function at the critical point is shown in Fig. 6. According to Eq. (11), $\overline{\ln C}$ should be proportional

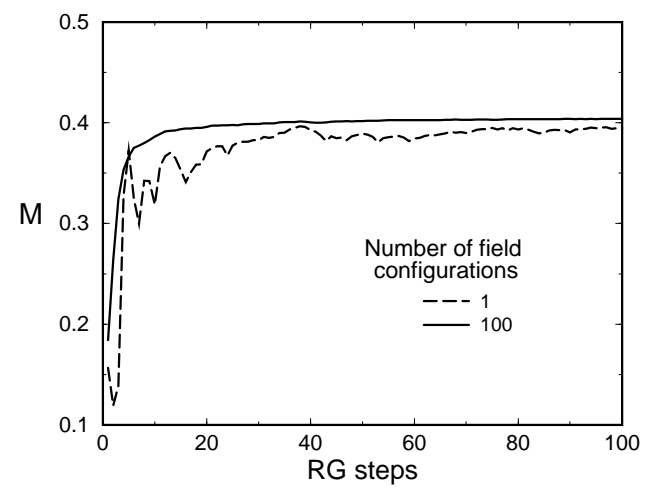

FIG. 5. The dependence of the inverse of the exponent $z$ on the distance from criticality. The solid line is the theoretical behavior of $1 / z$ for small $\delta$ values, according to Eq. (\$).

to the square root of $r$ for large $r$. The solid straight line is a fit to the data with $20 \leq r \leq 50$ and has a slope $-0.96 \pm 0.01$ and an intercept $0.52 \pm 0.01$. Our result is thus

$$
-\overline{\ln C}=0.96(1) r^{1 / 2}-0.52(1),
$$

where the figures in the parentheses denote the standard error.

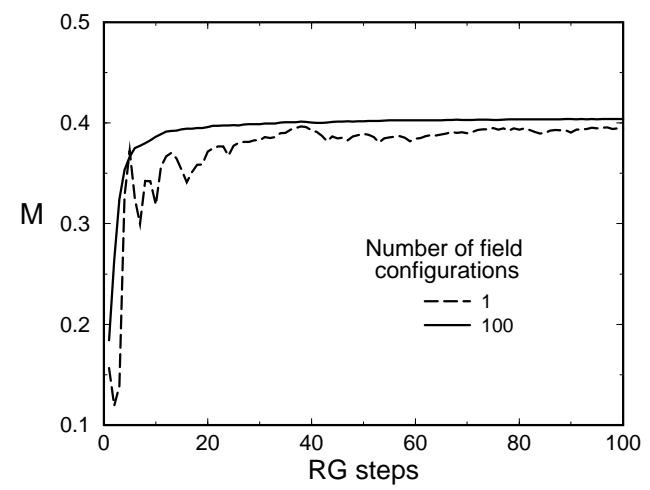

FIG. 6. The average of the logarithm of the correlation function at the critical point. The straight solid line is a fit to the data with $20 \leq r \leq 50$, the slope of $0.96 \pm 0.01$, and the intercept of $0.52 \pm 0.01$. The logarithm of the correlation function was averaged over 8000 random field configurations.

Our best scaling plot of the logarithm of the correlation function in the disordered phase is shown in Fig. ㅇ. The data for different $\delta$ collapse on a straight line, of slope $\approx-1$, when the exponent is $\tilde{\nu}=1.04$ which is slightly larger than the scaling value, predicted by Fisher $6 \tilde{\nu}=$ 1 , but smaller than the exponent determined by Young and Rieger 3 which obtained the best scaling at $\tilde{\nu}=1.1$. That the slope of the line is $\approx-1$ lends strong support to the suggestion by Fisher, mentioned in Ref. 3, that $\tilde{\xi}^{-1}=\overline{\ln h^{F}}-\overline{\ln J^{F}}$, which for the distribution (4) gives that $\tilde{\xi}^{-1}=\delta$ or $\ln \tilde{C}(r / \tilde{\xi}) \approx-\delta r$ for $r \gg \tilde{\xi}$. 


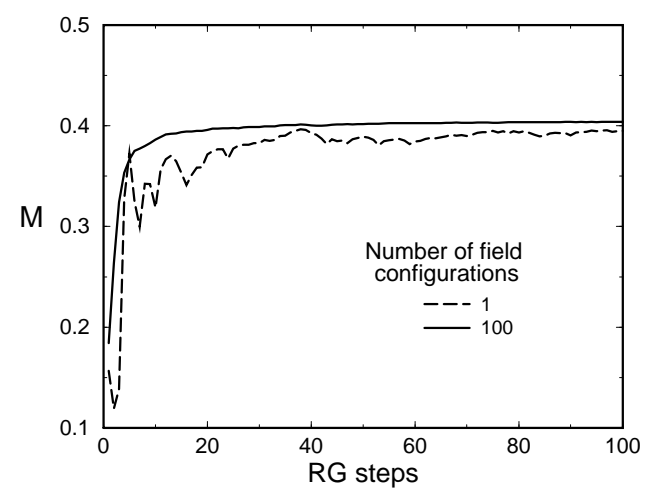

FIG. 7. A scaling plot of the average of the logarithm of the correlation function in the disordered phase with the exponent $\tilde{\nu}=1.04$.

\section{CONCLUSIONS}

In summary, we have analyzed some properties of the spin-1/2 quantum Ising model in a transverse random magnetic field (QIMRF) by means of the density-matrix renormalization group (DMRG). This method has two advantages with respect to other numerical methods. First of all, the computing time, for a given accuracy, scales linearly with the size of the system. Thus there is no severe limitation in increasing the size of the system. Our results show that typically the algorithm converges to the infinite-size limit after 50 - 100 DMRG steps, i.e., when the system is composed of $100-200$ sites. Second, an external uniform field in the $z$ direction could be explicitly added, allowing us to determine the scaling laws for the magnetization, both as a function of the external field and of the distance from criticality. This is the most relevant aspect of our approach. Indeed the introduction of the external field spoils the integrability which was exploited in Ref. 3, but is absolutely harmless from the point of view of the DMRG, the only effect being that a large number of random-field configurations is needed for a given accuracy.

It is evident that once the solvability of the model is not required, other systems, which cannot be mapped to free fermions, may equally well be investigated by means of the DMRG. The relevance of our results stands then not only in the accurate test of predicted scaling relations, and in the determination of nonuniversal coefficients and corrections beyond scaling, but mostly in the wide applicability of the method described above to the problem of quantum phase transitions in disordered systems. This is a relevant issue in solid-state physics. For instance, as we discussed in the Introduction, the QIMRF was obtained as an effective model for the Kondo lattice model.t Moreover, recent theoretical developments point out the relevance of quantum phase transitions 9 to the physics of high- $T_{c}$ superconductors. It has indeed been recently suggested that the properties of these systems close to optimal doping could be determined by the physics of nearby quantum critical point, associated to a magnetic 10 or an incommensurate charge-density-wave instability. 11 The anomalies observed in the optimally doped metallic phase and the behavior of the critical temperature have a natural interpretation once the lack of any characteristic energy scale beside the temperature itself is assumed as a consequence of the presence of a quantum critical point.

The approach described in this paper allows for a straightforward application to all those quantum systems that can be mapped onto effective spin models in the presence of random fields. Our results show indeed that the DMRG algorithm is a good and reliable alternative to other numerical methods within two respects: the possibility of studying systems of a considerable size, when needed, and the possibility of introducing an external field coupled to the order parameter, to extrapolate its behavior in the state of spontaneously broken symmetry.

\section{ACKNOWLEDGMENTS}

This work was supported by The Swedish Natural Science Research Council. S.C. acknowledges financial support from the Commission of the European Communities under HCM Contract No. ERBCHBGCT940724.

* Present address: Dipartimento di Fisica, Università degli Studi di Roma "La Sapienza," P. le A. Moro, 2 I-00185 Roma, Italy.

${ }^{1}$ G. Honner and M. Gulácsi, Phys. Rev. Lett. 78, 2180 (1997).

${ }^{2}$ S. Caprara and A. Rosengren, Europhys. Lett. 39 (1), 55 (1997).

3 A. P. Young and H. Rieger, Phys. Rev. B 53, 8486 (1996).

${ }^{4}$ B. M. McCoy and T. T. Wu, Phys. Rev. 176, 631 (1968).

${ }^{5}$ R. Shankar and G. Murthy, Phys. Rev. B 36, 536 (1987).

${ }^{6}$ D. S. Fisher, Phys. Rev. B 51, 6411 (1995).

${ }^{7}$ R. B. Griffiths, Phys. Rev. Lett. 23, 17 (1969).

${ }^{8}$ S. R. White, Phys. Rev. Lett. 69, 2863 (1992).

${ }^{9}$ J. A. Hertz, Phys. Rev. B 14, 1165 (1976).

${ }^{10}$ P. Monthoux, A. V. Balatsky, and D. Pines, Phys. Rev. B 46, 14803 (1992).

${ }^{11}$ C. Castellani, C. Di Castro, and M. Grilli, Phys. Rev. Lett. 75, 4650 (1995). 


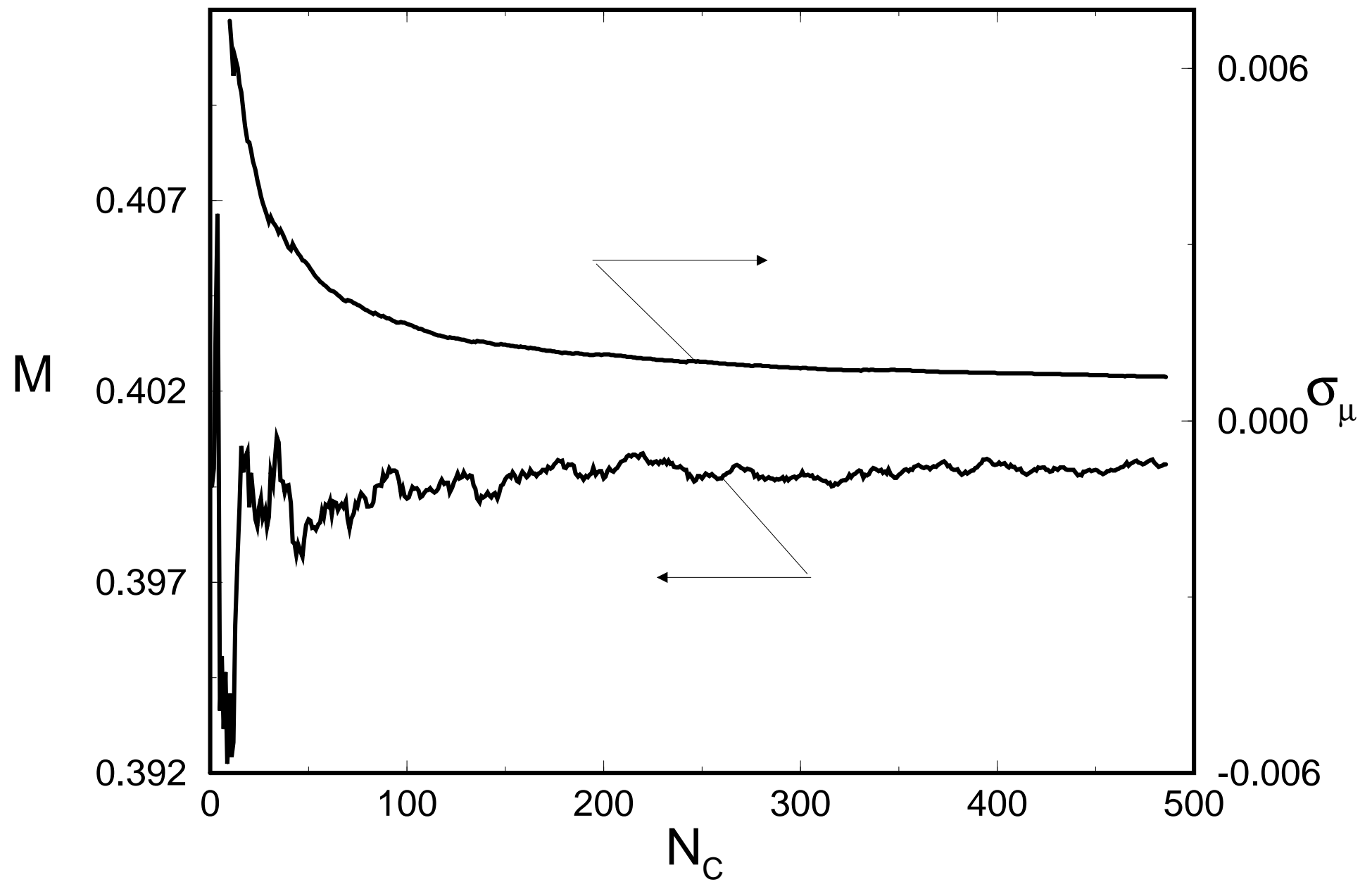




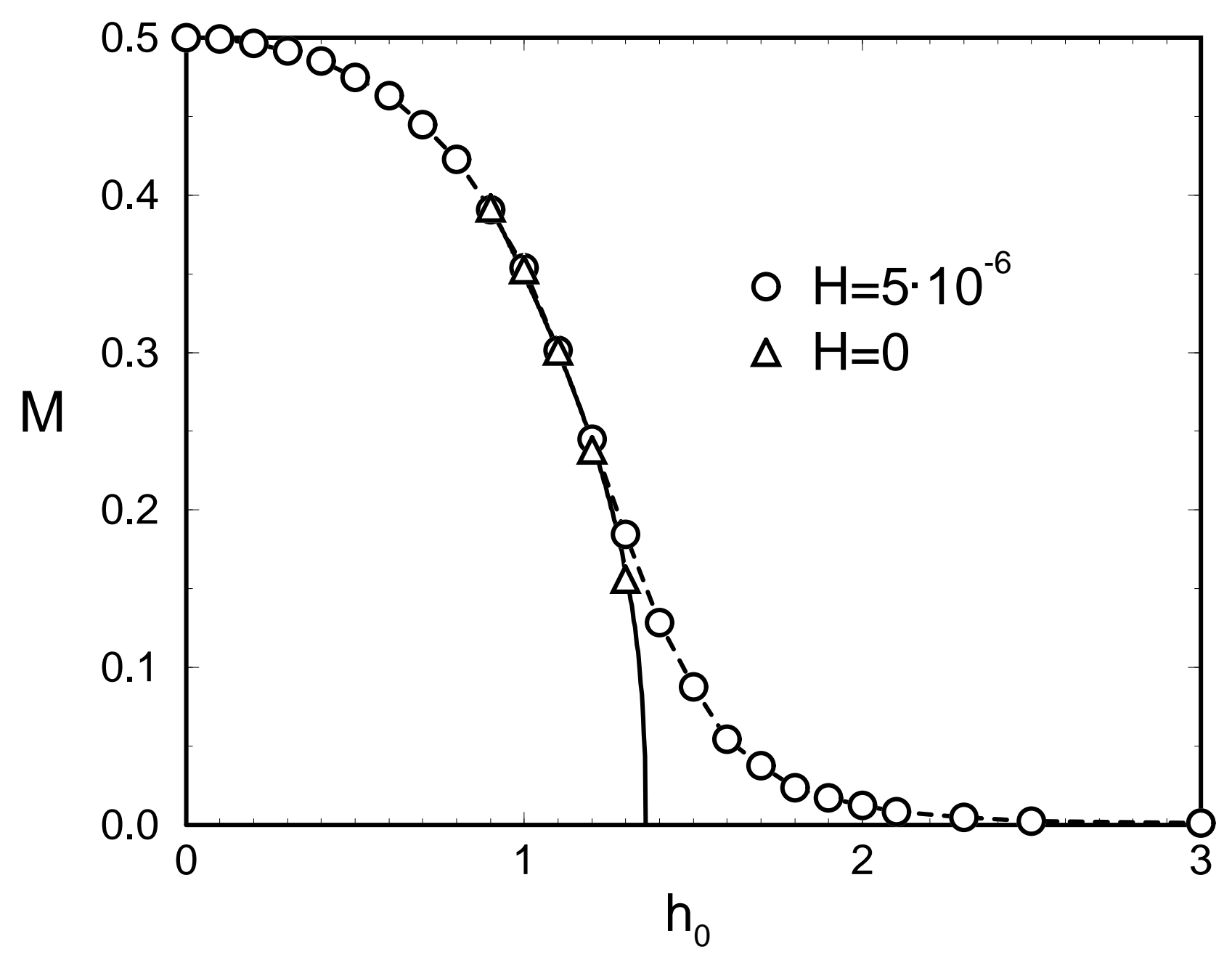




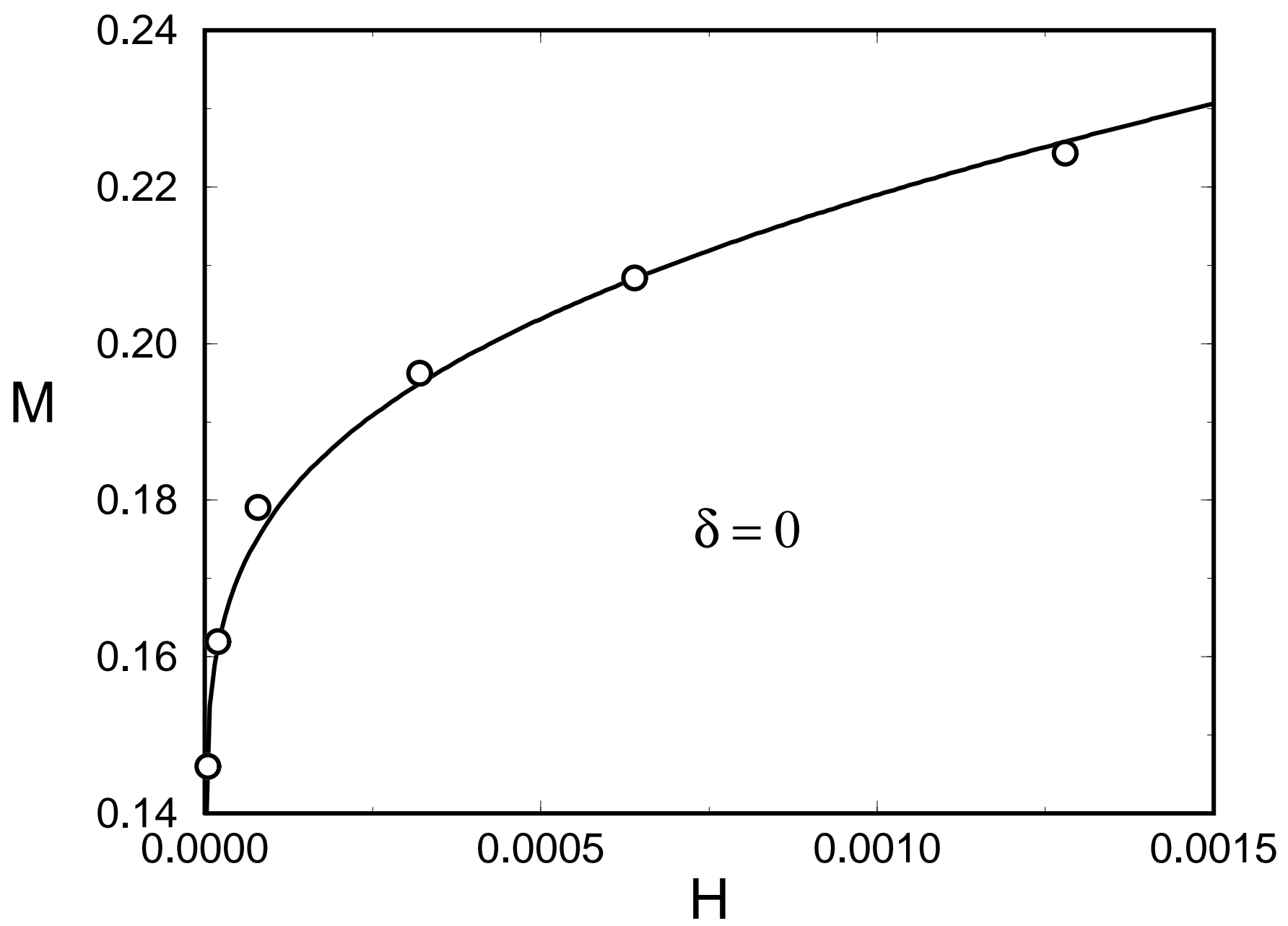




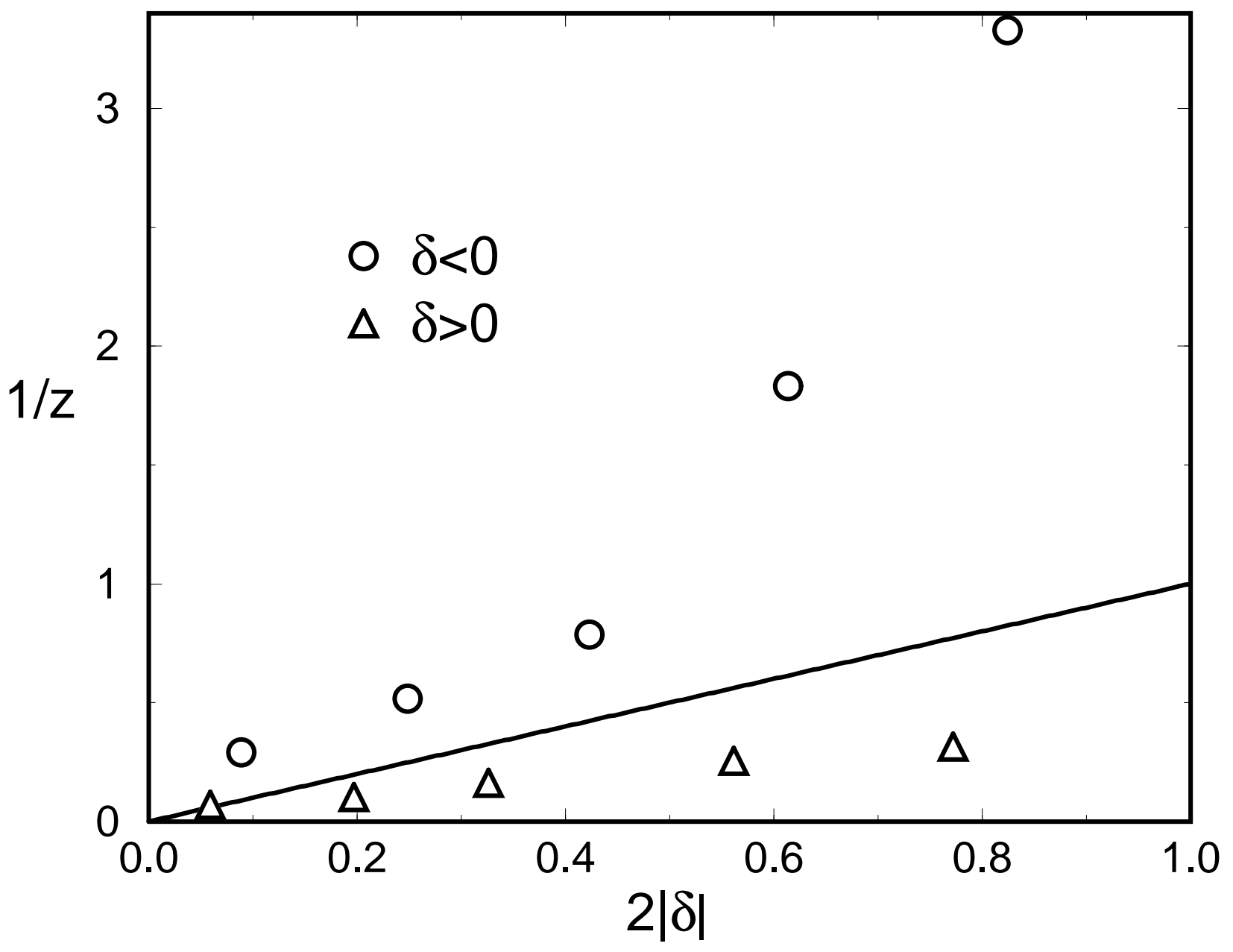




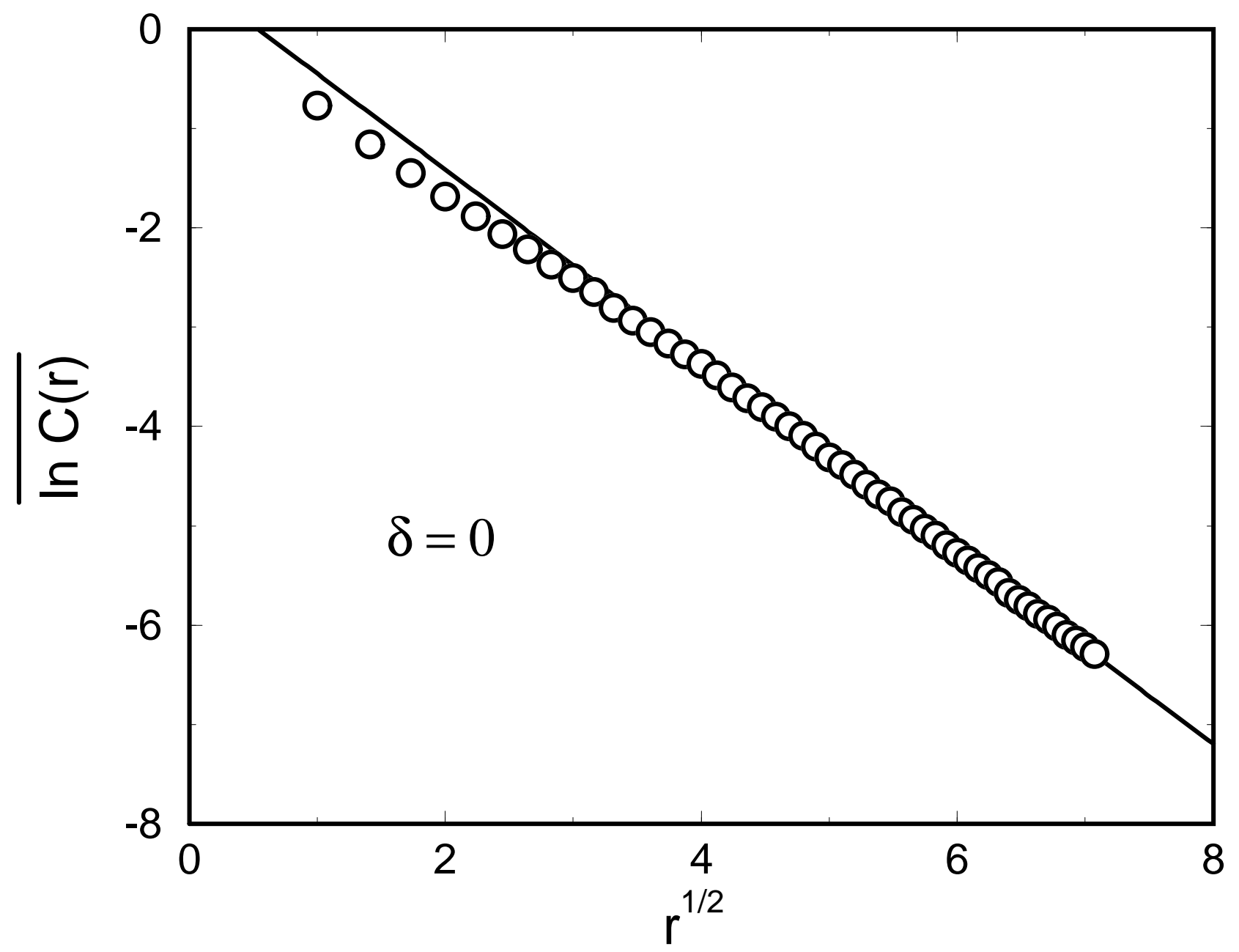




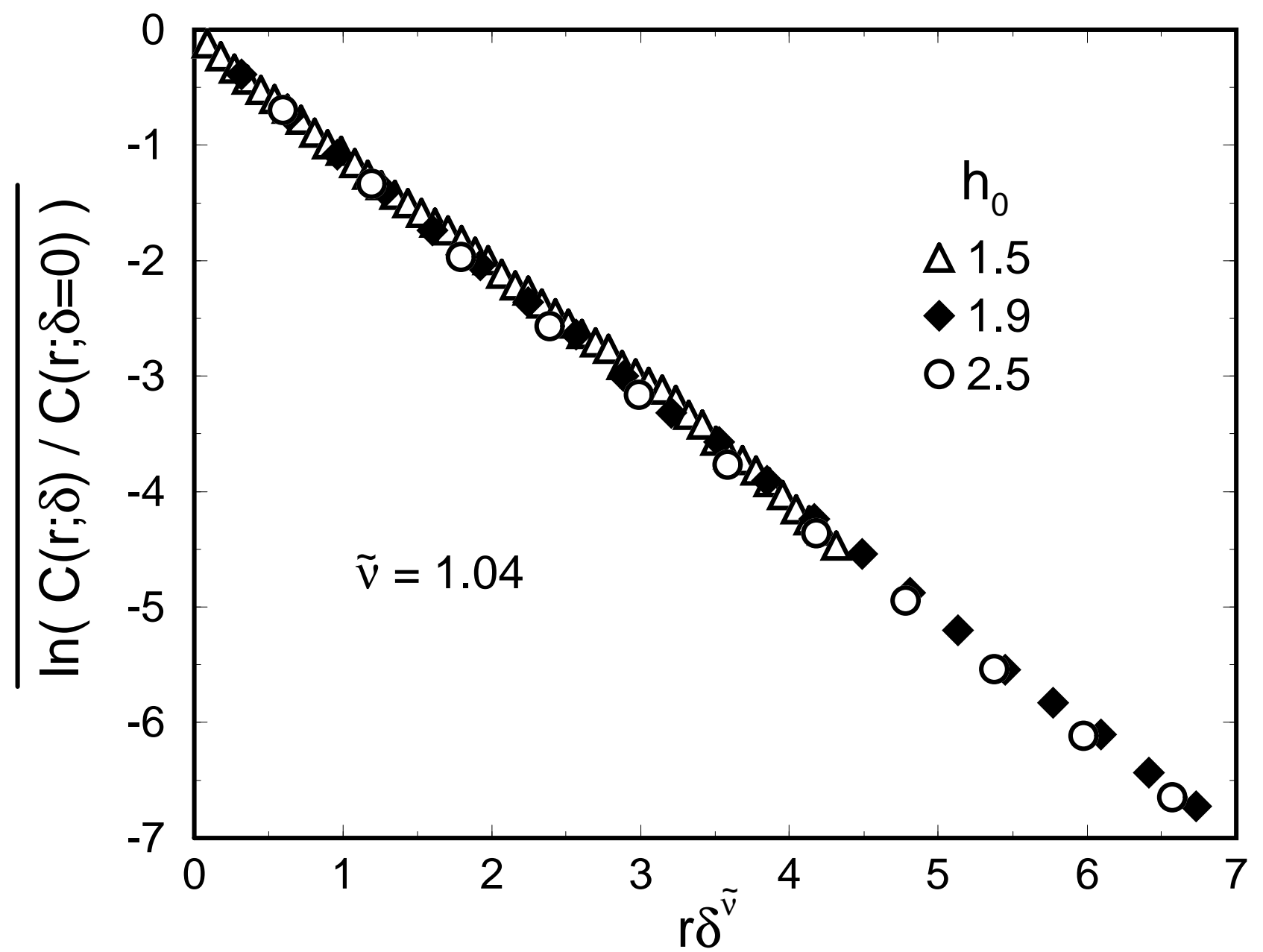

